\title{
BMJ Open Early vocational rehabilitation for people with spinal cord injury: a research protocol using realist synthesis and interviews to understand how and why it works
}

\author{
Jennifer Dunn (D) , ${ }^{1}$ Rachelle A Martin, ${ }^{2,3}$ Jonathan J Hackney, ${ }^{3}$ \\ Joanne L Nunnerley, ${ }^{1,3}$ Deborah Snell, ${ }^{1}$ John A Bourke, ${ }^{3,4}$ Andrew Hall, ${ }^{5}$ \\ Sarah Derrett ${ }^{6}$
}

To cite: Dunn J, Martin RA, Hackney JJ, et al. Early vocational rehabilitation for people with spinal cord injury: a research protocol using realist synthesis and interviews to understand how and why it works. BMJ Open 2021;11:e048753. doi:10.1136/ bmjopen-2021-048753

- Prepublication history and supplemental material for this paper is available online. To view these files, please visit the journal online (http://dx.doi. org/10.1136/bmjopen-2021048753).

Received 05 January 2021 Revised 17 March 2021 Accepted 18 March 2021
Check for updates

(C) Author(s) (or their employer(s)) 2021. Re-use permitted under CC BY-NC. No commercial re-use. See rights and permissions. Published by BMJ.

For numbered affiliations see end of article.

Correspondence to Dr Jennifer Dunn; jennifer.dunn@otago.ac.nz

\section{ABSTRACT}

Introduction Return to work after spinal cord injury (SCl) is linked to well-being and better physical and mental health outcomes. In New Zealand, work rates after SCI are lower than the general population. Vocational rehabilitation is one method of supporting return to work. Although the best model has not been determined, there is evidence supporting early intervention. However, most vocational rehabilitation research focuses on return to work outcomes without considering why vocational rehabilitation works, for whom and under what circumstances. Given this knowledge gap, we detail a realist synthesis protocol aiming to explain how contextual factors trigger relevant mechanisms to facilitate return to work after $\mathrm{SCl}$.

Methods and analysis This study will use a realist synthesis approach, following Realist And MEta-narrative Evidence Synthesis: Evolving Standards (RAMESES) guidelines. First, we will undertake a realist review of existing published and grey literature. Second, to assist with theoretical conceptualisation, we will interview people with SCI who have received vocational rehabilitation. Finally, we will survey people with $\mathrm{SCI}$ who received early vocational rehabilitation for theoretical testing and refinement.

Ethics and dissemination University of Otago Ethics Committee (Reference H19/170) has been obtained. A knowledge translation event will address issues relevant to wider implementation of the intervention and study findings. Findings will be also be disseminated through peer reviewed journals, conference presentations and formal reports.

\section{INTRODUCTION}

Work is important as it provides financial independence, can convey social standing, purpose, self-esteem ${ }^{1}$ and is a central component of identity. ${ }^{23}$ Return to work (RTW) for people with a new health condition is linked to increased well-being, quality of life and better physical and mental health outcomes. ${ }^{4-7}$ Furthermore, RTW encourages full societal
Strengths and limitations of this study

- A realist approach will allow for gaining a deeper understanding of how early vocational rehabilitation works, for it works best and the contexts that promote the activation of return to work.

- A realist approach facilitates the explanation of the complex nature of returning to work following an acquired neurological disability, making explicit the assumptions on how early vocational rehabilitation is expected to work and how contextual factors affect outcomes.

- The use of multiple data sources-interview and survey-alongside the realist literature review will enable the review to be grounded in the reality of healthcare delivery and address practice and policy changes.

Studies in only the English language will be included.

participation and independence, ${ }^{4}$ a feeling of 'normality' and productivity, ${ }^{8}$ and contributes to a country's economy. In New Zealand (NZ), unemployment rates for disabled people $(11.4 \%)$ are more than double the rate for non-disabled people $(4.5 \%) .^{9}$

Improving RTW rates for people with acquired disability is both important and complex. Fadyl and McPherson identified six factors which were important to workability: physical function; psychological function; thinking and problem solving skills; social and behavioural skills; the workplace; and factors outside the workplace. ${ }^{10}$ Having early optimistic perspectives and expectations of RTW, have also been recognised as important. ${ }^{11-14}$ In addition, accommodation by the employer, coworkers and the workplace are vital to assist disabled people back to work. ${ }^{15-21}$ One method of supporting RTW 
for people experiencing a new health condition is vocational rehabilitation. Vocational rehabilitation is defined as "whatever helps someone with a health problem to stay at, return to and remain in work: it is an idea and an approach as much as an intervention or a service. ${ }^{, 22}$

Fadyl and McPherson identified three broad models of vocational rehabilitation namely case coordination, programme based and supported employment. ${ }^{23}$ The best model of vocational rehabilitation has not been determined, although there is evidence supporting early intervention for people with a new health condition. ${ }^{22}{ }^{24}$ In the spinal cord injury (SCI) population, it has been suggested better vocational outcomes may be achieved if vocational rehabilitation is offered close to the time of initial injury before social disenfranchisement and reliance of disability benefits become ingrained. ${ }^{25}$ In NZ, one vocational service that incorporates a focus on early intervention is the NZ Spinal Trust (NZST) Vocational Rehabilitation Service (previously known as Kaleidoscope). This service is dedicated to returning people to meaningful and sustainable employment following SCI. It uses a 'client-centric' framework, which fosters hope for RTW very early after injury, often within the first weeks of admission to a rehabilitation unit. Specialist vocational consultants are based at the two spinal centres in NZ (Auckland and Christchurch) and provide early individual career coaching emphasising personalised education designed to promote empowerment. The service actively engages with employers early in a person's rehabilitation, to establish good relationships and facilitate a pathway back to previous employment where this is likely to be a realistic goal. If this is not realistic, the team focuses on early development of alternative RTW goals. It may be this focus on client-centred empowerment and early fostering of hope for RTW that sets this rehabilitation service apart from other vocational rehabilitation approaches. ${ }^{26}{ }^{27}$ A programme based on this model has recently been piloted in an Australian spinal unit, with results suggesting that implementing early vocational rehabilitation in the hospital setting has good potential for enhancing RTW following SCI. ${ }^{28}$

This protocol paper is presented in three sections. Section one presents the study aims and provides a rationale for using realist synthesis, section two describes our methodological steps for the realist synthesis and section three discusses how this synthesis protocol makes a novel contribution to knowledge. For the purpose of this study, RTW is defined as a return to paid employment among those who were in paid employment at the time of SCI, irrespective of the number of hours worked before or after SCI. Working age is defined as between 18 and 65 years of age (because in NZ $82 \%$ of children remain at secondary school until after their 17 th birthday ${ }^{29}$ and retirement age is 65 years).

This realist synthesis is conducted within a larger Health Research Council of NZ funded research project called EVocS (Early Vocational Rehabilitation following Neurological Disability Study; HRC 19-834). This current study aims to describe and understand how early intervention vocational rehabilitation services work and how the context in which it is provided impact on RTW outcomes among people with SCI. We aim to use this knowledge to develop an early intervention vocational rehabilitation implementation plan for improving RTW outcomes for people with other acquired impairments which can lead to disability, such as stroke.

\section{METHODS AND ANALYSIS}

\section{Rationale for using realist synthesis}

Realist synthesis is a type of literature review which aims to articulate theoretical understandings about how interventions work, and how people or systems respond (or not) to the resources available to them. ${ }^{30-33}$ Realist research seeks to develop deeper levels of explanation and understanding by recognising the importance of measuring outcomes, but also of explaining variations in outcomes and mediating mechanisms in different people or different circumstances. Mechanisms represent how intervention resources (ie, early intervention vocational rehabilitation interactions and activities) are received, interpreted and acted on by people or systems (ie, a person with SCI or the employer) to produce an outcome or pattern of outcomes. ${ }^{34}$ The realist inquiry seeks to explain examples of success, failure and various in-between outcomes. These theoretical explanations are referred to as middle range theories "which involve abstraction ... but are close enough to the observed data to be incorporated in propositions that permit empirical testing. ${ }^{, 35}$

Realist research as a whole acknowledges open and complex systems, recognising that one intervention (eg, with inputs that demonstrate high levels of fidelity) will not work in all situations. ${ }^{35}$ Realist approaches take the stance that, by understanding how and why interventions are expected to work, these 'inputs' can be adapted in response to the new context they are being delivered within so that outcomes are optimised. A realist approach to literature review contrasts with systematic reviews which primarily synthesise evidence to determine if an intervention is effective across a range of contexts, and the size of any intervention effect averaged across these different settings. Realist syntheses also draw on a heterogeneous evidence base, using an iterative approach to locate, review and then synthesise both grey and white literature reporting a variety of study designs. ${ }^{36}$

\section{METHODS}

Drawing on the standards and publication guidance for realist syntheses, ${ }^{35}{ }^{37}$ the work will be conducted in ten steps (figure 1). The project duration is 24 months with a completion date of 31 December 2021.

\section{Step 1: development of initial programme theory}

The primary aim of this realist synthesis is explanation building by exploring underlying theories and looking 


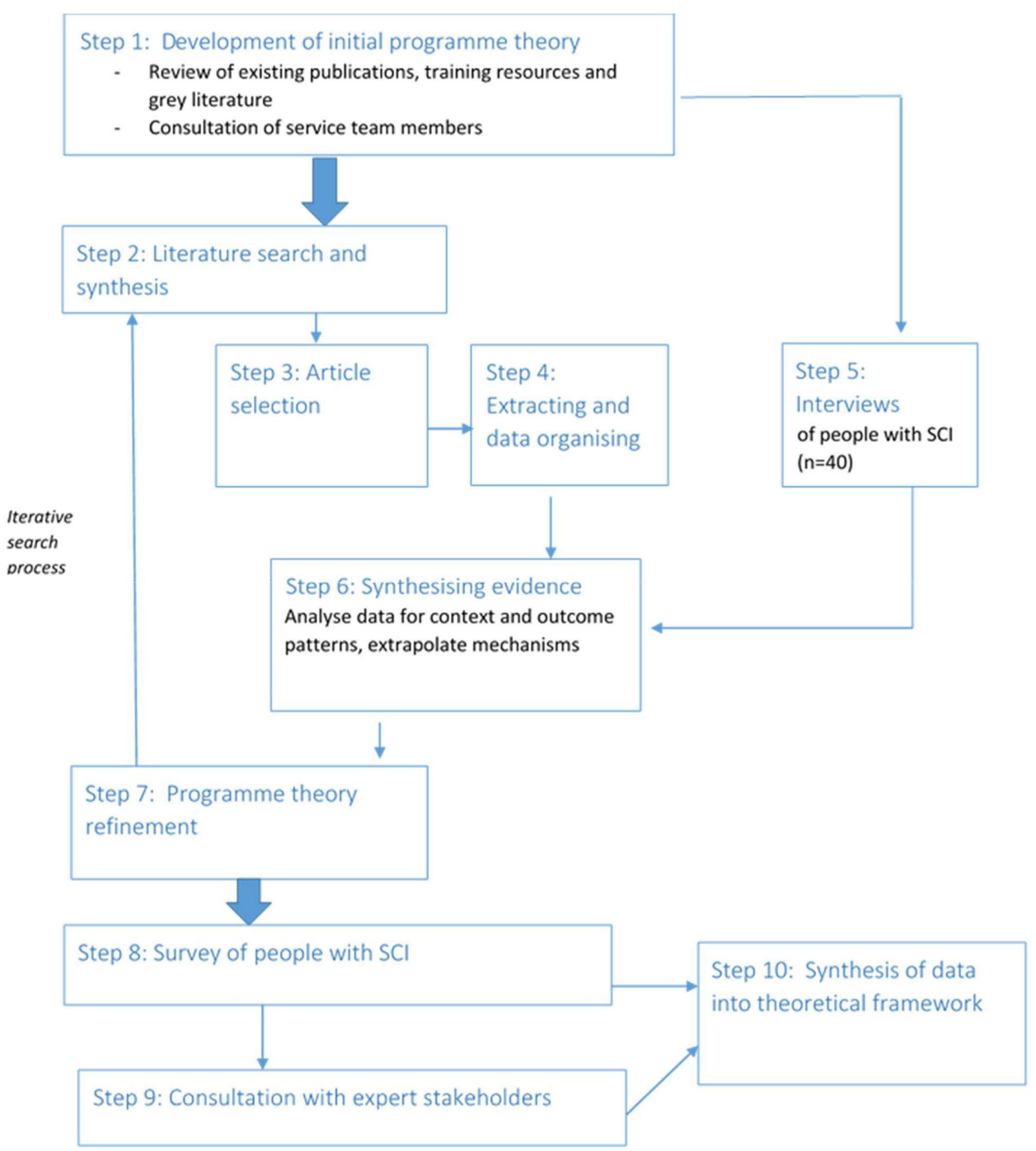

Figure 1 Flow chart of methodological steps. SCI, spinal cord injury.

at existing evidence to determine whether these theories are relevant. ${ }^{36}$ This initial programme theory will be a practical framework providing an early explanation of what is thought to work, how and under what conditions within delivery of vocational rehabilitation provided by the NZST Vocational Rehabilitation Service. Our ultimate goal is to construct provisional propositions that suggest how early intervention vocational rehabilitation might trigger mechanisms that either facilitate or hinder RTW following SCI. Using this initial programme theory, a list of possible context-mechanism-outcome (CMO) configurations will be generated to be tested and refined as we proceed with our realist synthesis. ${ }^{33}$ The CMO configurations are a heuristic device used within realist research, providing researchers with an analytic template to test hypothesised relationships between CMO.

To develop the initial programme theory a two-fold approach will be used: first, existing publications, training resources and grey literature will be reviewed and second, we will consult with staff members delivering the service.

(1): Review of literature: to build an initial understanding of the NZST Vocational Rehabilitation Service we will informally and rapidly search existing academic publications (such as peer reviewed articles) as well as grey literature (such as theses, reports, conference presentations, training resources and white papers). Search results will be reviewed and screened independently by JD and JJH. Articles that provide insights on how and why early intervention vocational rehabilitation affects RTW will be highlighted and retrieved for further review. Retrieved studies will be searched to identify propositions that have been developed for early intervention vocational rehabilitation, and for descriptions of potential mechanisms that determine the success or failure of early intervention vocational rehabilitation for people with illness or injury.

(2): Consultation with NZST vocational consultants: During the initial theory development we will also conduct face-to-face meetings with the vocational consultants working at the NZST to understand the processes they use to deliver early intervention vocational rehabilitation and to obtain their opinions and thoughts on the initial programme theory. 


\section{Step 2: literature search and synthesis}

Following the rapid literature search describe above, we will then perform a comprehensive search of the literature. Our search strategy for collecting evidence to test the developed initial theory will follow an iterative approach, an example of our search strategy for Medline is presented in online supplemental file 1 . We will perform an electronic data search for evidence that supports, refutes or refines our initial programme theory. A medical librarian, trained in realist reviews in the health sciences, will perform individual electronic searches in the following databases: Medline, Embase, PsycINFO, Emcare, Cinahl and Scopus.

A combination of subject headings and keywords will be searched and will broadly include the following: SCI, stroke, brain injuries, neurological rehabilitation, stroke rehabilitation, vocational rehabilitation, RTW, early intervention vocational rehabilitation, work preparation, work readiness, work reintegration. The populations included in the search will include stroke and traumatic brain injuries as similar barriers to employment as those associated with SCI have been identified. These barriers can be associated with pain and disability levels, depression and workplace factors. ${ }^{24}$ An international systematic review also suggests RTW rates are similar following both SCI and stroke. ${ }^{38}$ Boolean operators will be used to combine search terms to narrow, or broaden, the searches as required.

Realist synthesis approaches focus on theoretical development, and including a diversity of research designs providing more opportunities for in-depth theoretical explanations. ${ }^{3537}$ Therefore, we will include both quantitative and qualitative studies using a combination of publication types and keywords. To ensure the results remain manageable, we will limit the results to studies written in English. Since different types of research, reports, policy and guidance can be used to test and refine programme theories, we will also search for relevant grey literature by conducting internet-based searches. Reports and resources from other early intervention vocational rehabilitation programmes known within the research team and their networks will also be collated. We will also use reference list searching and citation tracking to identify additional white or grey literature that could contribute potentially relevant articles and author groups.

\section{Step 3: article selection}

Following the literature search, a first round of title and abstract screening will be conducted, followed by fulltext screening. Full-text screening will be independently reviewed by the two research team members and evaluated against the following criteria: (1) Does the study describe early vocational rehabilitation? (2) Is the study population inpatient at the time of receiving early vocational rehabilitation services? (3) Is the study population working age? (4) Does the study population have an acquired serious health condition? (5) Does the study describe RTW outcomes? Articles will be included if they meet either criteria one or 2 (or both), and all of criteria 3-5. Disagreements about articles to be included and excluded will be resolved through consensus between JD, JJH and RAM.

Unlike traditional systematic reviews where methodological quality of studies are appraised, realist reviews use a range of literature and documents to provide insight into the complex interventions that are being explored. In addition, very often realist reviews will only consider one element of a study to test a very specific question/ hypothesis about the link between context, mechanisms and outcome. As such a realist review does not use a preexisting quality checklist to judge the quality of the study. Instead a realist solution is to appraise the quality of articles for relevance and rigour and include those that meet these criteria. ${ }^{37} 39$ Evidence will therefore be appraised for relevance (does the article contribute to building or testing the theory) and rigour (are the methods used credible and trustworthy) by two team members (JD, JJH). A bespoke data extraction form will be used to record relevance and rigour judgements made of the extracted data, and will also include a brief description of the paper, bibliographic information and identification of possible $\mathrm{CMO}$ configurations as outlined in step 4 .

\section{Step 4: data extraction}

The aim of this step is to extract data that provides evidence of relationships between mechanisms, contexts and outcomes. Extraction of data from both peer-reviewed documents and grey literature will be conducted using QDA Miner 5, a qualitative and mixed-methods data analysis software package (Provalis Research). Two authors (JD and JJH) will independently read each article and extract data. Analytical tasks to be undertaken by the key review team members (JD, JJH and RAM) will include organising the extracted information into tables that support, refute or refine the initial programme theory; and looking for recurring patterns in the contexts, mechanisms and outcomes. In this way the initial programme theory will be iteratively reviewed and refined.

As the explanatory accounts are developed, additional literature searches will be undertaken to iteratively interrogate contextual factors, mechanisms, or existing theory emerging from the refined programme theory. To conduct these searches and collect additional evidence we will repeat steps $2,-4$.

\section{Step 5: interviews with people with SCI}

To further clarify and interrogate the relationships between the context in which early intervention rehabilitation services are provided, RTW outcomes, and possible mechanisms of intervention effect, we will also conduct interviews with people with SCI who have received early intervention vocational rehabilitation from the NZST Vocational Rehabilitation Service. By including the perspectives of people who received this service alongside the synthesis of literature we will iteratively refine the programme theory being developed. 
One-on-one interviews with approximately 40 people experiencing SCI who have received, or are receiving, early NZST Vocational Rehabilitation Services will be used to explore experiences of early vocational rehabilitation and various contexts and mechanisms that appear to contribute (or not) to the successful negotiation of RTW. Specifically, interviews will be conducted with the following groups of people with SCI:

1. Sustained 3-6 months previously (group 1).

2. Sustained 7-10 months previously (group 2).

3. Sustained 12-18 months previously (group 3).

These time frames were included as we felt it was important to explore the experiences of people who are currently receiving, or had recently received, input from the NZST Vocational Rehabilitation Services. The average length of stay at NZ spinal units following initial SCI is 74 days for a person with tetraplegia and 69 days for a person with paraplegia. ${ }^{40}$ The timeframes chosen allowed for those currently receiving vocational rehabilitation (group 1) as well as for those who had discharged from the spinal unit and would be in the process of exploring RTW options at varying time points following their injury (groups 2 and 3).

\section{Participant inclusion criteria}

1. NZ residents aged between 18 and 65 years at time of SCI.

2. Sustained an SCI resulting in admission to an NZ spinal unit for rehabilitation.

3. Employed at time of SCI.

4. Received early vocational rehabilitation by NZST.

5. Able to participate in interviews in the English language.

\section{Recruitment}

Potential participants will be purposively selected to ensure a representative sample and to specifically ensure that there are participants who have and have not RTW, participants with both traumatic and non-traumatic SCI, gender balance, participants who identify as Māori (the indigenous people of NZ), residing in rural and urban areas, and participants who have a comorbid (new or preexisting) mental health diagnosis. We feel that including people with comorbid mental health diagnosis is a strength of this research as it allows 'real-world' perspectives to be deliberately included.

The NZST delivers early vocational rehabilitation to approximately 85 people with newly acquired SCI (both traumatic and non-traumatic) per year when they are admitted to either of the two spinal units in NZ. Potential participants within the timeframes will be identified by NZST staff from their database of current clients and invited to participate in the interviews by an appropriate staff member. It is anticipated that $30-40$ participants with SCI will provide sufficient information to gain a preliminary understanding of the mechanisms and contextual factors that may impact the effectiveness of early vocational rehabilitation RTW outcomes.
While family or caregivers may be involved in interviews in a support role, it is not intended that their perspectives will be explicitly gained during this study. However, they will be asked for consent for any comments they do make to be transcribed and considered as part of the analysis.

\section{Data collection}

Following recruitment, participants will be interviewed (RM; experienced in both qualitative interviews and realist research methods). Interviews will be performed either online via a video-conferencing system, in the participant's own home or for group 1 participants in the spinal unit. It is anticipated that the interviews will take between 30 and $60 \mathrm{~min}$, depending on the participant's level of fatigue and ability to maintain attention. Interviews will be recorded on a digital recording device.

\section{Interview guide}

The interview guide will be developed based on the emerging theory (CMO configurations) and will explore how the proposed mechanisms explain (or not) the way early vocational rehabilitation works, and the contexts that promote (or not) the activation of mechanisms to achieve RTW. The questions will remain flexible with participants being asked to comment on activities and interactions during the provision of strategies or interventions that supported (or interfered with) RTW. Participant perspectives on the most effective way to optimise RTW following injury will also be sought.

Specific topics that interview questions will be based on include:

- What are the relationships between how early intervention services are provided, the contexts in which they are provided, and RTW for people with SCI who have received vocational rehabilitation services from the NZST?

- What positive and negative effects have been reported from people with SCI receiving early vocational intervention?

- What are the important contexts that determine whether the different mechanisms produce positive and negative effects, and why?

- Why does early vocational rehabilitation work better for some people than others?

Data from the interviews will be transcribed verbatim. All identifying information (ie, names and identifying characteristics) will be removed from the transcripts and no material which could identify participants will be used in any reports.

\section{Data analysis}

Interviews will be coded independently by two team members (RAM and JLN). Both will read and reread each interview transcript to gain a sense of the whole before manual line by line, with coding being undertaken independently. We will use NVivo to gather the data under the following a priori determined code headings: (1) intervention resources, actions or tasks, (2) contextual factors, 
(3) mechanisms and (4) outcomes. Team members will then compare and debate their coding decisions.

\section{Step 6: synthesising evidence}

Data synthesis of the interviews and the realist review will be undertaken by JD, JJH, RAM and JLN and synthesis results will be regularly discussed with the wider research team to ensure validity and consistency in the inferences made. Analytical tasks to be undertaken by the research team will include looking for recurring patterns in the $\mathrm{CMO}$ between the realist review and interview data. Throughout this synthesis, ongoing iterative literature searching will be performed to further explore emerging contextual factors, mechanisms, or existing theory emerging from the refined programme theory.

\section{Step 7: programme theory refinement}

Through synthesising findings between the various data sources, we will attempt to explain context-outcome patterns by identifying mechanisms that best explain the relationships within these patterns following the steps described by Wong et $a l^{37}$ Synthesis tasks will include linking the recurring patterns identified in previous steps to form explanatory accounts of how early intervention vocational rehabilitation works, for whom and under what circumstances. 'If-then-because' statements (ie, statements of the assumed context ['if'], outcome ['then']) and mechanism ['because'] will also be constructed to summarise the key CMO configurations. This will be an iterative process involving ongoing analysis, conceptualisation and refinement of theory. This refined programme theory will be used to develop the questions for the survey of past consumers of the service.

\section{Step 8: survey of past consumers of service}

A survey of clients who have received early vocational rehabilitation from the NZST between 1 January 2015 and 31 December 2019 will be undertaken.

\section{Inclusion criteria}

NZ citizen or resident who:

1. Sustained an SCI and discharged from BSU or ASRU between 1 January 2015 and 31 December 2019.

2. Employed at time of SCI.

3. eceived vocational rehabilitation services from NZST.

4. Able to read English and

5. Currently living in NZ.

\section{Recruitment}

Potential participants meeting the inclusion criteria will be identified from the NZST database by vocational consultants. Potential participants will be invited to participate in the survey by way of letter/email from NZST.

\section{Survey development}

Survey questions, developed by the research team, will reflect the elements and relationships between the programme theory emerging from previous phases of the project. Specifically, the survey will explore to what extent participants experience the key early vocational rehabilitation intervention mechanisms of effect as proposed by the theory. In addition, outcome data will include work status at specific time points post SCI and satisfaction with RTW status well as other variables determined by theory conceptualisation.

\section{Data collection}

Participants will be required to complete the survey either electronically via an emailed link or hard copy posted to them. All data will be collected and managed using Research Electronic Data Capture electronic data capture tools hosted at University of Otago, NZ. ${ }^{41}$

\section{Data analysis}

Data will be exported to SPSS version 25.0 for analysis. Analyses will be descriptive and used to check and refine the theory emerging from previous steps. In particular, we will use answers to the questions to check the relevance and applicability of emerging CMO configurations. This will allow development of an integrated and comprehensive theory informed by past research and grounded in the experiences of people with SCI who have received early vocational rehabilitation. This theory is therefore an analytical product and not merely a descriptive account.

\section{Step 9: consultation with expert stakeholders}

The emerging theory will be presented in a focus group with NZST staff in their capacity as expert stakeholders, to check its relevance. Staff will be presented with the key elements of the theory and will be asked to respond and reflect on the relevance and utility of the emerging theory in the SCI population. Data that support the articulated relationships of the emerging theory will be highlighted.

\section{Step 10: synthesis of data into theoretical framework}

Data from all sources will be synthesised and an early vocational rehabilitation programme theory articulated around CMO configurations will then be developed, which will allow for portability across other populations.

\section{Patient and public involvement}

As part of the development of this project, consultation with the Burwood Academy Consultation Network (https://www.burwood.org.nz/plex/bac-network/) a group of people with lived experience that review relevant research projects.

\section{ETHICS AND DISSEMINATION}

Ethical consideration include, but are not limited to, informed consent, anonymity and confidentiality, participant burden, reimbursement and the right to withdraw from the study. We aim to publish in peer-reviewed journals and present this work at relevant academic conferences. We will use existing relationships with consumer groups within NZ, such as the NZST and the Burwood Academy of Independent Living to disseminate lay reports to stakeholders and people with SCI. The theoretical explanations proposed as a result of this research will generate testable propositions about how 
early vocational rehabilitation works for people with SCI. It is anticipated that this refined programme theory can then be used to develop an early intervention vocational rehabilitation implementation plans for improving RTW outcomes for people with other acquired impairments, such a stroke. We will hold a knowledge translation workshop presenting the findings of this research with the aim of exploring how best to implement this intervention in different contexts/populations and thus identifying any additional resources required to support this.

\section{Author affiliations}

${ }^{1}$ Department of Orthopaedic Surgery and Musculoskeletal Medicine, University of Otago, Christchurch, New Zealand

${ }^{2}$ Department of Medicine, University of Otago, Wellington, New Zealand

${ }^{3}$ Burwood Academy of Independent Living, Christchurch, New Zealand

${ }^{4}$ Menzies Health Institute, Griffith University, Gold Coast, Queensland, Australia

${ }^{5}$ New Zealand Spinal Trust, Christchurch, New Zealand

${ }^{6}$ Preventative and Social Medicine, University of Otago, Dunedin, New Zealand

Contributors Conceptualisation: JD, RAM, JLN, DS, SD; methodology: JD, RAM; formal analysis: JD, RAM, JJH, JLN, DS; data curation: JD, RAM, JJH, JLN, DS, JAB, SD; investigation JD, JJH, RAM, DS, JAB; writing original draft JD; writing review and editing JD, RAM, JJH, JLN, DS, JAB, AH, SD; funding acquisition JD; all authors read and approved the final manuscript.

Funding This work is supported by the Health Research Council of New Zealand and Ministry of Social Development (HRC 19-834).

Competing interests All authors have completed the ICMJE uniform disclosure form at www.icmje.org/coi_disclosure.pdf and declare: all authors had financial support from Health Research Council of New Zealand for the submitted work; AH is employed by the NZ Spinal Trust.

Patient consent for publication Not required.

Provenance and peer review Not commissioned; externally peer reviewed.

Supplemental material This content has been supplied by the author(s). It has not been vetted by BMJ Publishing Group Limited (BMJ) and may not have been peer-reviewed. Any opinions or recommendations discussed are solely those of the author(s) and are not endorsed by BMJ. BMJ disclaims all liability and responsibility arising from any reliance placed on the content. Where the content includes any translated material, BMJ does not warrant the accuracy and reliability of the translations (including but not limited to local regulations, clinical guidelines, terminology, drug names and drug dosages), and is not responsible for any error and/or omissions arising from translation and adaptation or otherwise.

Open access This is an open access article distributed in accordance with the Creative Commons Attribution Non Commercial (CC BY-NC 4.0) license, which permits others to distribute, remix, adapt, build upon this work non-commercially, and license their derivative works on different terms, provided the original work is properly cited, appropriate credit is given, any changes made indicated, and the use is non-commercial. See: http://creativecommons.org/licenses/by-nc/4.0/.

ORCID iD

Jennifer Dunn http://orcid.org/0000-0002-2894-7533

\section{REFERENCES}

1 Dunn DS, Brody C. Defining the good life following acquired physical disability. Rehabil Psychol 2008;53:413-25.

2 Watson TJ. Sociology, Work and Industry [electronic resource]. Routledge, 2008.

3 Oppermann JD. Interpreting the meaning individuals ascribe to returning to work after traumatic brain injury: a qualitative approach. Brain Inj 2004;18:941-55.

4 Waddell G, Burton AK. Is work good for your health and well-being? London, UK: The Stationery Office, 2006.

5 Yasuda S, Wehman P, Targett P, et al. Return to work after spinal cord injury: a review of recent research. NeuroRehabilitation 2002;17:177-86.
6 The Royal Australasian College of Physicians. Australasian faculty of occupational and environmental medicine position statement on realising the health benefits work. Sydney, 2011.

7 Giaquinto S, Ring H. Return to work in selected disabilities. Disabil Rehabil 2007;29:1313-6.

8 Johansson U, Tham K. The meaning of work after acquired brain injury. Am J Occup Ther 2006;60:60-9.

9 Statistics New Zealand. Labour force survey. Wellington, 2017.

10 Fadyl JK, McPherson KM, Schlüter PJ, et al. Factors contributing to work-ability for injured workers: literature review and comparison with available measures. Disabil Rehabil 2010;32:1173-83.

11 Chan SKK, Man DWK. Barriers to returning to work for people with spinal cord injuries: a focus group study. Work 2005;25:325-32.

12 Schönherr MC, Groothoff JW, Mulder GA, et al. Vocational reintegration following spinal cord injury: expectations, participation and interventions. Spinal Cord 2004;42:177-84.

13 van Velzen JM, van Bennekom CAM, van Dormolen M, et al. Factors influencing return to work experienced by people with acquired brain injury: a qualitative research study. Disabil Rehabil 2011;33:2237-46.

14 Murphy GC, Young AE, Brown DJ, et al. Explaining labor force status following spinal cord injury: the contribution of psychological variables. J Rehabil Med 2003;35:276-83.

15 Colella A. Coworker distributive fairness judgments of the workplace accommodation of employees with disabilities. Acad Manage Rev 2001;26:100-16.

16 MacEachen E, Kosny A, Ferrier S, et al. The "toxic dose" of system problems: why some injured workers don't return to work as expected. J Occup Rehabil 2010;20:349-66.

17 Schoppen T, Boonstra A, Groothoff JW, et al. Employment status, job characteristics, and work-related health experience of people with a lower limb amputation in the Netherlands. Arch Phys Med Rehabil 2001;82:239-45.

18 Schoppen T, Boonstra A, Groothoff JW, et al. Factors related to successful job reintegration of people with a lower limb amputation. Arch Phys Med Rehabil 2001;82:1425-31.

19 Dunstan DA, MacEachen E. Bearing the brunt: co-workers experiences of work reintegration processes. J Occup Rehabil 2013;23:44-54.

20 Tjulin A, Maceachen E, Ekberg K. Exploring workplace actors experiences of the social organization of return-to-work. $J$ Occup Rehabil 2010;20:311-21.

21 Nunnerley J, Dunn J, McPherson K, et al. Return to work for severely injured survivors of the Christchurch earthquake: influences in the first 2 years. Disabil Rehabil 2016;38:987-93.

22 Waddell G, Burton AK, Kendall AS. Vocational rehabilitation what works, for whom, and when? London, 2008.

23 Fadyl JK, McPherson KM. Approaches to vocational rehabilitation after traumatic brain injury: a review of the evidence. $J$ Head Trauma Rehabil 2009;24:195-212.

24 Cancelliere C, Donovan J, Stochkendahl MJ, et al. Factors affecting return to work after injury or illness: best evidence synthesis of systematic reviews. Chiropr Man Therap 2016;24:32.

25 Ottomanelli L, Goetz LL, Suris A, et al. Effectiveness of supported employment for veterans with spinal cord injuries: results from a randomized multisite study. Arch Phys Med Rehabil 2012;93:740-7.

26 Kelly M. Early and community vocational rehabilitation model (poster). Australian and New Zealand spinal cord Society conference. Brisbane: Building Bridges, 2017.

27 Kelly M. A discourse of hope and possibility [plenary]. Proceedings of the New Zealand Occupational Health Conference 2014.

28 Middleton JW, Johnston D, Murphy G, et al. Early access to vocational rehabilitation for spinal cord injury inpatients. J Rehabil Med 2015;47:626-31.

29 Ministry of Education. Retention of students in senior secondary school. Wellington, 2020.

30 Pawson R, Tilley N. Realistic evaluation. Sage, 1997.

31 Salter KL, Kothari A. Using realist evaluation to open the black box of knowledge translation: a state-of-the-art review. Implement Sci 2014;9:115.

32 Astbury B, Leeuw FL. Unpacking black boxes: mechanisms and theory building in evaluation. Am J Eval 2010;31:363-81.

33 Emmel N, Greenhalgh J, Manzano A. Doing realist research. Sage, 2018.

34 Dalkin SM, Greenhalgh J, Jones D, et al. What's in a mechanism? Development of a key concept in realist evaluation. Implement Sci 2015;10:49.

35 Wong G, Greenhalgh T, Westhorp G, et al. RAMESES publication standards: realist syntheses. BMC Med 2013;11:21.

36 Pawson R, Greenhalgh T, Harvey G, et al. Realist review-a new method of systematic review designed for complex policy interventions. J Health Serv Res Policy 2005;10:21-34. 
37 Wong G, Westhorp G, Greenhalgh J, et al. Quality and reporting standards, resources, training materials and information for realist evaluation: the RAMESES II project. Health Serv Deliv Res 2017:5:1-108.

38 van Velzen JM, van Bennekom CAM, Edelaar MJA, et al. How many people return to work after acquired brain injury?: a systematic review. Brain Inj 2009;23:473-88.

39 Pawson R, Greenhalgh T, Harvey G. Realist synthesis: an introduction. Manchester: ESRC Research Methods Programme, University of Manchester, 2004.
40 New Zealand Spinal Cord Injury Registry. New Zealand spinal cord injury registry annual summary report 2018, 2020.

41 Harris PA, Taylor R, Minor BL, et al. The REDCap consortium: building an international community of software platform partners. J Biomed Inform 2019;95:103208.

42 Harris PA, Taylor R, Thielke R, et al. Research electronic data capture (REDCap) - a metadata-driven methodology and workflow process for providing translational research informatics support. J Biomed Inform 2009;42:377-81. 